\title{
Computer simulations of liquid silica: equation of state and liquid-liquid phase transition
}

\author{
Ivan Saika-Voivod, ${ }^{1}$ Francesco Sciortino, ${ }^{2}$ and Peter H. Poole ${ }^{1}$ \\ ${ }^{1}$ Department of Applied Mathematics, University of Western Ontario, London, Ontario N6A 5B7, Canada \\ ${ }^{2}$ Dipartimento di Fisica and Istituto Nazionale per la Fisica della Materia, \\ Universita' di Roma La Sapienza, Piazzale Aldo Moro 2, I-00185, Roma, Italy
}

(Dated: October 25, 2018)

\begin{abstract}
We conduct extensive molecular dynamics computer simulations of two models for liquid silica [the model of Woodcock, Angell and Cheeseman, J. Phys. Chem. 65, 1565 (1976); and that of van Beest, Kramer and van Santen, Phys. Rev. Lett. 64, 1955, (1990)] to determine their thermodynamic properties at low temperature $T$ across a wide density range. We find for both models a wide range of states in which isochores of the potential energy $U$ are a linear function of $T^{3 / 5}$, as recently proposed for simple liquids [Rosenfeld and P. Tarazona, Mol. Phys. 95, 141 (1998)]. We exploit this behavior to fit an accurate equation of state to our thermodynamic data. Extrapolation of this equation of state to low $T$ predicts the occurrence of a liquid-liquid phase transition for both models. We conduct simulations in the region of the predicted phase transition, and confirm its existence by direct observation of phase separating droplets of atoms with distinct local density and coordination environments.
\end{abstract}

PACS numbers: 65.50.+m 64.30.+t, 64.60.My, 64.70.Ja

\section{INTRODUCTION}

First order liquid-liquid phase separation, in which two liquids of distinct chemical composition coexist, are common in multicomponent systems. However, there has in recent years been a growing interest in first order liquidliquid phase transitions that occur without a change of composition, but rather with a change in density $\rho$ as temperature $T$ or pressure $P$ is varied. Experimental evidence for the occurrence of such transitions has been found for a wide range of systems, including $\mathrm{Si}$ [1, 2 , I, Se, S 3, 4], $\mathrm{Al}_{2} \mathrm{O}_{3}-\mathrm{Y}_{2} \mathrm{O}_{3}$ melts [5], $\mathrm{C}$ [6], $\mathrm{H}_{2} \mathrm{O}$ [7, 8], and $\mathrm{P}$ [9]. Liquid-liquid transitions have also been observed in molecular dynamics (MD) computer simulations of $\mathrm{Si}$ [10, 11], $\mathrm{H}_{2} \mathrm{O}$ [12, 13] and $\mathrm{C}$ 14]. Theoretical studies have long predicted liquid-liquid transitions for a variety of model fluids; for examples, see Refs. [15, 16, 17, 18, 19, 20, 21, 22, 23, 24].

In the case of water, the proposed liquid-liquid phase transition occurs in the supercooled liquid, i.e. for $T$ less than that of the melting line [7]. Closely associated with the possibility of a liquid-liquid transition in supercooled water is the phenomenon of polyamorphism in the amorphous solid occurring below the glass transition temperature, $T_{g}$. Polyamorphism refers to the occurrence of distinct amorphous solid forms of a material [25, 26. In the most prominent cases of polyamorphism, such as water [27, 28, 29], an abrupt first-order-like transition occurs from a low-density form to a distinct high-density form as the amorphous material is compressed at low $T$. For water it was proposed that the observed polyamorphism of the amorphous solid is a sub- $T_{g}$ manifestation of the thermodynamic instability associated with the liquidliquid phase transition [12].

Computer simulation studies of the ST2 water model [30] support this view of the relationship between liquid-liquid phase transitions and polyamorphism. The qualitative features of water polyamorphism are clearly displayed in simulations of ST2 water [31]. Correspondingly, the critical temperature $T_{c}$ marking the onset of liquid-liquid phase separation has been determined [13], as well as a characteristic pattern of thermodynamic "precursors" for $T>T_{c}$ consistent with the instability at $T=T_{c}$ [32]. These precursors include the occurrence of a density maximum and a compressibility maximum. Simulation studies of the thermodynamic properties of two other water-like models, TIP4P [33] and SPC/E [34], find the same pattern of thermodynamic precursors observed for ST2 for $T>T_{c}$ 32, 35, and also display polyamorphism in simulations of the amorphous solid. However, in these systems, simulations of the liquid at lower $T$, to test for the onset of a liquid-liquid phase transition, have not yet been attempted due to prohibitively long equilibration times.

Substances that are structurally similar to water, such as $\mathrm{Si}, \mathrm{Ge}, \mathrm{GeO}_{2}$, and $\mathrm{SiO}_{2}$ (silica), have the potential to exhibit similar behavior [36]. Particular attention has been paid to silica because of its technological and geological importance. Polyamorphism is indeed observed in compression experiments on amorphous silica [37, 38, 39], and is also qualitatively reproduced in computer simulations 40, 41, 42. Though not as dramatic as is found for amorphous solid water, the polyamorphism of silica may also be due to a trend toward liquid-liquid phase separation 443, 44. Indeed, liquid state simulations of the silica model of Woodcock, Angell and Cheeseman (denoted here "WAC silica") 450 have shown that the same pattern of thermodynamic precursors of the liquid-liquid phase transition found in water simulations also occurs in this system 443. However, as in TIP4P and SPC/E water, the low $T$ simulations required to test for an explicit liquid-liquid phase transition in WAC silica have 
not been attempted to date.

At the same time, recent advances concerning the properties of liquids at and below the melting line are expanding our ability to study the states where liquid-liquid phase transitions may occur. Of particular importance is the recent prediction [46] that an isochore of the potential energy $U$ should be a linear function of $T^{3 / 5}$ for a simple, cold, dense liquid. Since this relation is proposed to be valid in the limit of low $T$, it is a useful relation for studying the properties of a deeply supercooled liquid. Notably, several recent works showed that this prediction is obeyed at low $T$ for binary LennardJones liquids 47, 48, 49]. This observation provided a physical basis for extrapolating $U$ (and thermodynamic properties derived from it) to $T$ near $T_{g}$, and so made possible determination of the Kauzmann temperature for this system.

In this paper, we examine in detail the behavior of two simulation models of silica: (i) WAC silica; and (ii) the widely used potential of van Beest, Kramer and van Santen [50], denoted here as "BKS silica." Our goal is to determine if either model displays a liquid-liquid phase transition. We find that our computer simulation data for the thermodynamic properties of these silica models obey the prediction of Ref. 46] over a wide range of $T$ and $V$. We exploit this result to construct equations of state for BKS and WAC silica, and find that liquidliquid phase transitions are predicted for both models at low $T$. We then conduct simulations near the predicted phase transition, and confirm the occurrence of liquidliquid phase transitions for both BKS and WAC silica by direct observation.

\section{MOLECULAR DYNAMICS SIMULATIONS}

The calculations presented here for WAC silica are based on the data set generated for Ref. 43. These simulations consisted of $N=450$ atoms (300 O, $150 \mathrm{Si}$ atoms) and were conducted in the constant $(N, V, E)$ ensemble. ( $E$ is the total internal energy, $V$ is the volume.) The effects of electrostatic interactions were incorporated using the Ewald summation technique [51].

For the BKS model, we conduct new simulations of a system of $N=1332$ atoms. As for WAC silica, Ewald summations are used to include electrostatic interactions. The Ewald parameter ( $\alpha$ in the notation of Ref. [51]) is fixed to $2.5 \mathrm{~nm}^{-1}$ for all state points simulated. For each state point, the system is equilibrated to near the desired $T$ using periodic velocity rescaling. All averages are reported for constant $(N, V, E)$ simulations that follow the equilibration stage. In all cases, averages are evaluated over a time that is at least ten times longer than the average time required for an $\mathrm{Si}$ atom to diffuse $0.2 \mathrm{~nm}$.

The BKS potential has the unphysical feature that the interaction energy of a $\mathrm{Si}$ and $\mathrm{O}$ atom pair diverges to $-\infty$ as their separation goes to 0 . Though not a problem at ambient $T$ and $P$, this feature will occasionally manifest itself at high $T$ and $P$. We have added a short range term to the BKS potential that prevents this from occurring, but which does not alter the form of the BKS potential at larger separations 52 .

The $(V, T)$ coordinates of the state points simulated

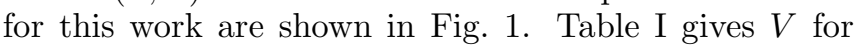
each of the isochores studied.

\section{TEMPERATURE DEPENDENCE OF POTENTIAL ENERGY ISOCHORES}

Ref. [46] predicts that the isochoric $T$ dependence of the potential energy $U$ of a simple, dense, cold liquid is given by,

$$
U=a+b T^{3 / 5}
$$

where $a$ and $b$ are constants for a given $V$.

Here we test if Eq. 1 is obeyed by WAC and BKS silica. We plot isochores of $U$ against $T^{3 / 5}$ and fit a straight line to the data (Fig. 2). At all $V$ studied, we find that Eq. 1 fits the data well. Consistent with the prediction of Ref. 46], the best fits occur for the smallest $V$, and the quality of the fits decreases somewhat as $V$ increases. For BKS silica, we find that Eq. 1 fits to all isochores within numerical uncertainty. For WAC silica, most of the data available to us fits Eq. 1 within numerical uncertainty. However, for the largest $V$, systematic deviations from Eq. 11 occur if all WAC data up to the highest $T$ are included. Yet even for these large $V$ isochores, the WAC data are consistent with an approach to the behavior of Eq. 11 at low T. By excluding several of the highest $T$ data points, shown as open circles in Fig. 1, we recover a fit within numerical uncertainty even for the largest $V$ isochores for WAC silica.

The $V$ dependence of the fit parameters $a$ and $b$ so obtained for both models is shown in Fig. 3. The success of the fits to Eq. 1 over a wide range of $T$ and $V$, and the smooth variation of $a$ and $b$ with $V$ show that the predictions of Ref. 46 appear to be valid for silica, a "complex" liquid with anisotropic molecular interactions, at least in the limits of low $T$ and low $V$.

In addition, it is important to note the $V$ dependence of $a$. $a$ provides an estimate of $U$ in the limit $T \rightarrow 0$, which classically is coincident with the limit as $T \rightarrow 0$ of $A$, the Helmholtz free energy. For both WAC and BKS silica we find a range of $V$ in which the curvature of the $a$ versus $V$ curve is negative. In this range, the condition for thermodynamic stability for a single phase, $\left(\partial^{2} A / \partial^{2} V\right)_{T}>0$, is not satisfied [53], suggesting that both WAC and BKS silica would undergo a liquid-liquid phase separation at low $T$, if not preempted by crystallization or vitrification. 


\section{MODEL EQUATION OF STATE}

Having identified a region of validity for Eq. 1, we can use this relation to construct a representation of the thermodynamic properties of WAC and BKS silica in terms of a continuous function of $T$ and $V$. The model equations of state so generated will allow us to clarify the liquidliquid phase separation suggested by the $V$ dependence of $a$ in Fig. 3 .

We first fit polynomials to the $V$ dependence of $a(V) \simeq$ $\sum_{n=0}^{4} \alpha_{n} V^{n}$ and $b(V) \simeq \sum_{n=0}^{4} \beta_{n} V^{n}$, to obtain a functional representation of $U$ :

$$
U(V, T)=a(V)+b(V) T^{3 / 5} .
$$

The coefficients $\alpha_{n}$ and $\beta_{n}$ are given in Table II. The internal energy is $E=U+U_{k}$; for the classical ionic models of silica considered here, the kinetic energy is $U_{k}=\frac{9}{2} R T$, where $R$ is the gas constant. Hence, our model for $E$ is,

$$
E(V, T)=a(V)+b(V) T^{3 / 5}+\frac{9}{2} R T .
$$

Next, we seek a functional representation of the entropy $S(V, T)$, so that a model for $A(V, T)$ can be obtained from $A=E-T S . S$ at arbitrary $V$ and $T$, relative to the entropy $S\left(V_{0}, T_{0}\right)$ of a reference state, can be evaluated by thermodynamic integration [53. We carry this out in two steps, first along an isotherm, and then along an isochore.

We compute the change $\Delta S_{T}=S\left(V, T_{0}\right)-S\left(V_{0}, T_{0}\right)$ along an isotherm $T=T_{0}$ using $\Delta A=\Delta E-T_{0} \Delta S_{T}$, where $\Delta A=A\left(V, T_{0}\right)-A\left(V_{0}, T_{0}\right)$ and $\Delta E=E\left(V, T_{0}\right)-$ $E\left(V_{0}, T_{0}\right) . \Delta E$ is evaluated from Eq. 3. $\Delta A$ is a difference due to a volume change from $V_{0}$ to $V$ at $T=T_{0}$, and is found from an isothermal integration of $d A=-P d V$; that is,

$$
\Delta A=-\int_{V_{0}}^{V} P\left(V^{\prime}, T_{0}\right) d V^{\prime} .
$$

To obtain a functional representation for $\Delta A$ therefore requires one for $P(V)$ at $T=T_{0}$. We obtain the required data from our MD simulations and fit to them a polynomial $P \simeq \sum_{n=0}^{5} \gamma_{n} \rho^{n}$, where the density $\rho=1 / V$ (Fig. A). The coefficients $\gamma_{n}$ are given in Table III, along with the choices of $T_{0}$ for WAC and BKS silica. Using this polynomial model for $P\left(V, T_{0}\right)$, the integration in Eq. 6 yields an expression for $\Delta A$ which combined with that for $\Delta E$, gives a model function for $\Delta S_{T}$ [54. In terms of $E$ and $P$, the expression for $\Delta S_{T}$ is,

$$
\Delta S_{T}=\frac{1}{T_{0}}\left[E\left(V, T_{0}\right)-E\left(V_{0}, T_{0}\right)+\int_{V_{0}}^{V} P\left(V^{\prime}, T_{0}\right) d V^{\prime}\right] .
$$

The change $\Delta S_{V}=S(V, T)-S\left(V, T_{0}\right)$ is the entropy difference at fixed $V$ due to a temperature change from $T_{0}$ to $T$. This we find from an isochoric integration of,

$$
d S=\frac{d E}{T}=\frac{1}{T}\left(\frac{\partial E}{\partial T}\right)_{V} d T .
$$

That is,

$$
\Delta S_{V}=\int_{T_{0}}^{T} \frac{1}{T^{\prime}}\left(\frac{\partial E}{\partial T^{\prime}}\right)_{V} d T^{\prime}
$$

This evaluation is carried out using our representation of $E(V, T)$ in Eq. 2.

Combining the contributions of both isothermal and isochoric changes, $S$ at an arbitrary state point is given by,

$$
S(V, T)=S\left(V_{0}, T_{0}\right)+\Delta S_{T}+\Delta S_{V} .
$$

Using the model functions for $E$ and $S$, we thus obtain a function modeling $A(V, T)$. The equation of state $P(V, T)$ is found from,

$$
P(V, T)=-\left(\frac{\partial A}{\partial V}\right)_{T} .
$$

Note that the resulting expression for $P(V, T)$ does not contain the unknown reference entropy $S\left(V_{0}, T_{0}\right)$ since this constant disappears after the differentiation in Eq. 9.

To summarize, we construct a model $P(V, T)$ equation of state using as input, polynomial fits of (i) the $V$ dependence of $a$ and $b$, and (ii) one reference isotherm of $P$. As a check of this equation of state, we compare in Fig. 5 isochores of $P$ versus $T$, evaluated directly from simulation, and as calculated from the above modeling procedure.

\section{THERMODYNAMIC BEHAVIOR OF WAC AND BKS SILICA}

For the description of the thermodynamic properties of tetrahedrally coordinated liquids such as silica or water, it is useful to determine the location and shape of curves in the space of $P, V$ and $T$ at which specific thermodynamic conditions are met. In the present context, three such curves are important:

(i) Along the "temperature of maximum density" (TMD) line the condition,

$$
\left(\frac{\partial P}{\partial T}\right)_{V}=0
$$

is satisfied [36]. At such a point, an isobar of $\rho$ as a function of $T$ is a maximum, and at lower $T, \rho$ decreases as $T$ decreases. The presence of a TMD line is a hallmark of liquids in which local tetrahedral order is prominent, and is observed experimentally in silica, as well as in water.

(ii) The metastability limit of the liquid, or spinodal line, is defined by [36],

$$
\left(\frac{\partial P}{\partial V}\right)_{T}=0
$$


(iii) Along the " $K_{T}^{\max }$ line," the isothermal compressibility $K_{T}$ is a maximum with respect to $V$ at constant $T$. It is found by locating points satisfying,

$$
\left(\frac{\partial K_{T}}{\partial V}\right)_{T}=0
$$

where,

$$
K_{T}=-\frac{1}{V}\left(\frac{\partial V}{\partial P}\right)_{T},
$$

and then checking to confirm that the extremum so identified is a maximum [36, 55].

Spinodal lines are necessarily associated with a second order critical point that terminates a line of first order phase transitions [36]. When a such a critical point occurs at $T=T_{c}$, a $K_{T}^{\max }$ line will emanate from the critical point for $T>T_{c}$. However, the occurrence of a $K_{T}^{\max }$ line does not imply the occurrence of a critical point at lower $T$; i.e. the occurrence of a $K_{T}^{\max }$ line is a necessary, but not a sufficient condition, for the occurrence of a critical point 55.

The locations of these lines are shown in Fig. 1 projected onto the $V-T$ plane; and in Fig. 6, projected onto the $T-P$ plane. We find that the pattern of behavior revealed is qualitatively the same both for WAC and BKS silica, and that this is the same pattern found also from water simulations employing the ST2, TIP4P [32] and $\mathrm{SPC} / \mathrm{E}$ models 35 .

Most significant is the occurrence both for WAC and BKS silica, of a spinodal line in the low $T$ liquid regime that is distinct from the liquid-gas spinodal boundary. This spinodal is the metastability boundary associated with a liquid-liquid phase transition. As $T \rightarrow 0$ it coincides with the points of inflection in the $a$ versus $V$ curves of Fig. 3. The critical point of this liquid-liquid phase transition occurs at the point of maximum $T$ on the spinodal line.

\section{LIQUID-LIQUID PHASE SEPARATION}

If the spinodal curves predicted by the equations of state developed in Section IV are correct, and if we can conduct equilibrium simulations in the unstable regions so identified, we should observe characteristic signs of phase separation. When a liquid-liquid phase separation occurs in a constant- $V$ simulation such as employed here, both phases coexist within the simulation box, each in its own distinct region, separated by an interface. Each phase will have a distinct bulk density as well as a distinct local structure.

We therefore carry out new MD runs for both WAC and BKS silica at state points that approach the spinodal curve associated with the liquid-liquid phase transition. These state points are identified as squares in Fig. 11. To facilitate comparison of BKS and WAC silica, we simulate a system of $N=750$ atoms for both. The lowest $T$ state simulated for each model $(T=2000 \mathrm{~K}$ for $\mathrm{BKS}, T=4000 \mathrm{~K}$ for WAC) is near the predicted critical point. Because of the low $T$, we are are unable to bring these lowest $T$ states fully into equilibrium; however, the results obtained do serve to establish the trend in the behavior at the lowest $T$.

To test for the occurrence of two phases with distinct local structure, we examine the local coordination environment of the silicon atoms. We consider $g(r)$, the $\mathrm{Si}-\mathrm{Si}$ radial distribution function (RDF); $4 \pi r^{2} g(r) d r$ is the probability that a $\mathrm{Si}$ atom will be found at a distance between $r$ and $r+d r$ of a reference $\mathrm{Si}$ atom. We decompose $g(r)$ according to the contributions made by successive nearest neighbors (nn) of a given $\mathrm{Si}$ atom, labelled in ascending order of distance from an $\mathrm{Si}$ atom. That is, we define sub-RDF's $g_{i}(r)$ according to

$$
g(r)=\sum_{i=1}^{\infty} g_{i}(r)
$$

where $4 \pi r^{2} g_{i}(r) d r$ is the probability that the $i$ th nearest neighbor of a randomly selected $\mathrm{Si}$ atom will be found at a distance between $r$ and $r+d r$.

Fig 7 shows $g_{5}(r)$ for BKS and WAC silica for several different $T$ along the isochores indicated by open squares in Fig. 11. For $T$ above $T_{c}, g_{5}(r)$ is a unimodal function of $r$. As $T$ decreases, the width of the $g_{5}(r)$ distribution increases. That is, rather than finding a more sharply defined 5 th nn coordination environment as $T$ decreases, the distribution of locations of 5th nn's becomes broader. For $T$ near $T_{c}, g_{5}(r)$ becomes bimodal. This behavior shows that two distinct populations of 5 th $\mathrm{nn}$ coordination environments are emerging in the liquid as $T$ decreases. Similar behavior is observed for $g_{6}(r)$, and more weakly in $g_{7}(r)$ and $g_{8}(r)$. This is in contrast to the behavior of $g_{1}(r)$ through $g_{4}(r)$ which simply become sharper and narrower distributions with decreasing $T$ (not shown).

If the emergence of these distinct coordination environments corresponds to the onset of liquid-liquid phase separation, we should find that Si atoms with similar coordination environments are spatially correlated. That is, there should be relatively compact droplets of the two distinct phases.

To test for this, we require systems of larger spatial extent than the $N=750$ atom simulations used to calculate $g_{5}(r)$. We therefore initiate simulations of a system of $N=6000$ atoms for the lowest $T$ states where phase separation should be most prominent $(T=2000 \mathrm{~K}$ for $\mathrm{BKS}, T=4000 \mathrm{~K}$ for WAC). As above, excessive equilibration times prevent us from bringing these states fully into equilibrium, and so we only use these simulations to establish the trend in behavior. We note that an incompletely equilibrated system should underestimate the amount of phase separation, since droplets (if present) will have had less time to form and grow.

We examine snapshots of these $N=6000$ atom BKS and WAC configurations. The minimum occurring between the two peaks of $g_{5}(r)$ for the lowest $T$ in Fig. 7 
provides a convenient threshold $r^{*}$ for partitioning the $\mathrm{Si}$ atoms into two populations according to their 5th nn coordination. We show in Fig. 8 snapshots of the positions of all the Si atoms in the BKS and WAC systems. Light spheres are $\mathrm{Si}$ atoms having their 5th nn at a distance greater than $r^{*}$; these atoms have a low-density coordination environment, compared to the average. Dark spheres are $\mathrm{Si}$ atoms having their $5 \mathrm{th} \mathrm{nn}$ at a distance less than $r^{*}$; these atoms have a high-density coordination environment. Spatially correlated droplets of atoms with the same type of coordination environment are readily visible, for both WAC and BKS silica. This is consistent with the occurrence of liquid-liquid phase separation for both of these silica models.

\section{DISCUSSION}

The two principal conclusions of the present work are:

(i) For two silica models, BKS and WAC, there exists a wide range of $T$ and $V$ within which isochores of $U$ for the liquid phase conform to Eq. 1. This occurs in spite of the fact that the prediction of Ref. [46] was made for simple liquids, and not for liquids with non-trivial local structure such as occurs in silica. This result suggests that the physical basis for Eq. 1 is quite robust and provides a valuable tool for probing the low $T$ properties of a wide range of liquid systems.

(ii) The model equation of state constructed by exploiting Eq. 1 predicts the occurrence of a liquid-liquid phase transition in BKS and WAC silica. We confirm the presence of this transition by direct simulations near the predicted critical point. Thus BKS and WAC silica join the rank of simulation models for tetrahedrally coordinated liquids in which a liquid-liquid phase transition has been directly observed. Whether or not such a phase transition occurs in real liquid silica (see below), it's presence in the behavior of BKS silica is important to note, since this model is currently in wide use for simulation studies of silica under a variety of conditions.

Given the common behavior found for BKS and WAC silica, it is appropriate to inquire whether we should therefore expect to find the same pattern of thermodynamic behavior, including a liquid-liquid phase transition, in real silica; or whether these two models share a common flaw that makes them unrealistic in this respect. To attempt to address this, we seek a basis for comparing the behavior of the BKS and WAC models with each other and with other tetrahedral liquids, both simulated and real. To proceed we choose as a scaling temperature $T^{*}$ the highest value of $T$ reached along the TMD line.

As shown in Table III, the ratio $T_{c} / T^{*}$ is approximately 0.4 for both WAC and BKS silica. Assuming that this ratio is also valid for real silica gives $T_{c}=730 \mathrm{~K}$, a temperature well below $T_{g}$ for silica at $1450 \mathrm{~K}$. Hence, if the WAC and BKS models are representative of the thermodynamic properties of silica, then we should not expect to directly observe an equilibrium liquid-liquid phase tran- sition in supercooled liquid silica.

This is in contrast to the case of water. The ST2 model gives $T_{c} / T^{*}=0.80$, implying that $T_{c}=194 \mathrm{~K}$ for real water. This is well above $T_{g}=136 \mathrm{~K}$ for water, though as yet still outside the easily accessible experimental range. (See however, Ref. [7].)

The present analysis is consistent with recent interpretations [32, 43, 44] of the phenomenology of polyamorphism as observed in amorphous solid silica and water. In this interpretation, the manifestation of polyamorphism for a system where the liquid phase exhibits the tendency toward a liquid-liquid phase transition at low $T$ will depend on the relationship of $T_{g}$ to $T_{c}$. When $T_{c}>T_{g}$, as may be the case for water, polyamorphic behavior in the amorphous solid will be prominent, with a large and sharp density increase observed when the glass in compressed. When $T_{c}<T_{g}$, behavior characteristic of polyamorphism will be weaker, though not necessarily absent, as is the case for silica.

However, the precise nature of the behavior to be expected when $T_{c}<T_{g}$ remains an open area of research. For $T<T_{g}$, a standard application of equilibrium thermodynamics is not appropriate and so predictions based on the model equations of state presented here do not apply. Nonetheless, new thermodynamic approaches, based on a separation of the configurational degrees of freedom (frozen in at $T_{g}$ ) and vibrational degrees of freedom (which are always in thermal equilibrium) may be employed to derive a free energy expression that can be used used to locate the (cooling-rate dependent) location of the critical point [56, 57]. In this approach, a negative curvature of the $V$ dependence of $U$ along the Kauzmann line would be sufficient to guarantee the presence of an instability in the glassy phase.

We also note that the value of $T$ for the $K_{T}^{\max }$ line at atmospheric $P$ is about $80 \%$ of $T^{*}$ for both WAC and BKS silica (see Fig. 6). Applying this ratio to real silica suggests that a maximum of $K_{T}$ (or, equivalently, a minimum of the bulk modulus) could occur when liquid silica is compressed isothermally at $T$ just above, but near $T_{g}$. This raises the possibility to directly observe a $K_{T}^{\max }$ line in experiments of supercooled liquid silica under pressure. Confirming or refuting this prediction would be an important step in establishing the applicability of the pattern of thermodynamic behavior presented here, to real silica and other tetrahedral liquids.

\section{Acknowledgments}

We are grateful to G. Parisi for useful discussions. ISV and PHP wish to thank NSERC (Canada) for financial support, and the Dipartimento di Fisica and Istituto Nazionale per la Fisica della Materia, Universita' di Roma La Sapienza, for their financial support and hospitality during visits when portions of this work were carried out. FS acknowledges financial support from INFM-PRA-HOP and PRIN98. Substantial computing 
[1] M.O. Thompson, G.J. Galvin, J.W. Mayer, P.S. Peercy, J.M. Poate, D.C Jacobson, A.G. Cullis and N.G. Chew, Phys. Rev. Lett. 52, 2360 (1984).

[2] E.P. Donovan, F. Saepen, D. Turnbull, J.M. Poate, and D.C. Jacobson, J. Appl. Phys. 57, 1795 (1985).

[3] V.V. Brazhkin, R.N. Voloshin, S.V. Popova and A.G. Umnov, Phys. Lett. A 154, 413 (1991).

[4] V.V. Brazhkin, S.V. Popova, R.N. Voloshin and A.G. Umnov, High Pressure Research 6, 363 (1992).

[5] S. Aasland and P.F. McMillan, Nature 369, 633 (1994).

[6] M. Togaya, Phys. Rev. Lett. 79, 2474 (1997).

[7] O. Mishima and H.E. Stanley, Nature 396, 329 (1998).

[8] O. Mishima, Phys. Rev. Lett. 85, 334 (2000).

[9] Y. Katayama, T. Mizutani, W. Utsumi, O. Shimomura, M. Yamakata and K.-I. Funakoshi, Nature 403, 170 (2000).

[10] W.D. Ludke and U. Landman, Phys. Rev. B 37, 4656 (1988)

[11] C.A. Angell, S. Borick and M. Grabow, J. Non-Cryst. Solids 205-207, 463 (1996).

[12] P.H. Poole, F. Sciortino, U. Essmann and H. E. Stanley, Nature 360, 324 (1992).

[13] S. Harrington, R. Zhang, P. H. Poole, F. Sciortino and H. E. Stanley, Phys. Rev. Lett. 78, 2409 (1997).

[14] J.N. Glosli and F.H. Ree, Phys. Rev. Lett. 82, 4659 (1999).

[15] P.C. Hemmer and G. Stell, Phys. Rev. Lett. 24, 1284 (1970).

[16] A.C. Mitus, A.Z. Patashinskii and B.I. Shumilo, Phys. Lett. 113A, 41 (1985).

[17] E.G. Ponyatovsky and I.O. Barkalov, Materials Science Reports 8, 147 (1992).

[18] S. Sastry, F. Sciortino and H.E. Stanley, J. Chem. Phys. 98, 9863, (1993).

[19] P.H. Poole, F. Sciortino, T. Grande, H.E. Stanley and C.A. Angell, Phys. Rev. Lett. 73, 1632 (1994).

[20] C. J. Roberts, A. Z. Panagiotopoulos and Pablo G. Debenedetti, Phys. Rev. Lett. 77, 4386 (1996).

[21] C.F. Tejero and Marc Baus, Phys. Rev. E 57, 4821 (1998).

[22] E.A. Jagla, J. Chem. Phys. 111, 8980 (1999).

[23] G. Franzese, et al., preprint, cond-mat/0005184.

[24] J.A. Jagla, preprint, cond-mat/0006381.

[25] G.H. Wolf, S. Wang, C.A. Herbst, D.J. Durben, W.J. Oliver, Z.C. Kang, and C. Halvorsen, in High-Pressure Research: Application to Earth and Planetary Sciences, edited by Y.S. Manghnani and M.H. Manghnani (American Geophysical Union, Washington, 1992) p. 503.

[26] P.H. Poole, T. Grande, C.A. Angell and P.F. McMillan Science 275, 322 (1997).

[27] O. Mishima, L.D. Calvert and E. Whalley, Nature 314, 76 (1985).

[28] O. Mishima, K. Takemura and K. Aoki, Science 254, 406 (1991).

[29] O. Mishima, J. Chem. Phys. 100, 5910 (1994).

[30] F.H. Stillinger and A. Rahman, J. Chem. Phys. 60, 1545 (1974).

[31] P.H. Poole, U. Essmann, F. Sciortino and H. E. Stanley,
Phys. Rev. E 48, 4605 (1993).

[32] F. Sciortino, P.H. Poole, U. Essmann, and H.E. Stanley, Phys. Rev. E 55, 727 (1997).

[33] W.L. Jorgensen, J. Chandrasekhar, J. Madura, R.W. Impey and M. Klein, J. Chem. Phys. 79, 926 (1983).

[34] H.J.C.Berendsen, J.P.M.Postma, W.F.Van Gunsteren, A.Dinola, J.R.Haak, J. Chem. Phys. 81, 3684 (1984)

[35] S. Harrington, P. H. Poole, F. Sciortino and H. E. Stanley, J. Chem. Phys. 107, 7443 (1997)

[36] P.G. Debenedetti, Metastable Liquids: Concepts and Principles, Princeton University Press, Princeton (1998).

[37] H. Sugiura, K.-I. Kondo and A. Sawaoka in HighPressure Research in Geophysics (eds S. Akimoto and M.H. Manghnani) 551 (Reidel, Dordrecht, 1982)

[38] M. Grimsditch, Phys. Rev. Lett. 52, 2379 (1984).

[39] R. J. Hemley, H. K. Mao, P. M. Bell, and B. O. Mysen Phys. Rev. Lett. 57, 747 (1986).

[40] M.S. Somayazulu, et al. J. Phys.: Condens. Matter 5, 6345 (1993).

[41] J.S. Tse, D.D. Klug, and Y. LePage, Phys. Rev. B 46, 5933 (1992).

[42] W. Jin, R.K. Kalia, P. Vashishta and J.P. Rino, Phys. Rev. Lett. 71, 3146 (1993).

[43] P.H. Poole, M. Hemmati and C.A. Angell Phys. Rev. Lett. 79, 2281 (1997).

[44] D.L. Jacks, Phys. Rev. Lett. 84, 4629 (2000).

[45] L.V. Woodcock, C.A. Angell and P.A. Cheeseman, J. Chem. Phys. 65, 1565 (1976).

[46] Y. Rosenfeld and P. Tarazona, Mol. Phys. 95, 141 (1998).

[47] F. Sciortino, W. Kob and P. Tartaglia, Phys. Rev. Lett. 83, 3214 (1999).

[48] B. Coluzzi, Ph.D Thesis, University of Roma La Sapienza (1999); B. Coluzzi, P. Verrocchio and G. Parisi, Phys. Rev. Lett. 84, 306 (2000)

[49] S. Sastry, Phys. Rev. Lett. 85, 590 (2000).

[50] B.W.H. van Beest, G.J. Kramer, and R.A. van Santen, Phys. Rev. Lett. 64, 1955 (1990).

[51] M.P. Allen and D.J. Tildesley, Computer Simulation of Liquids (Oxford University Press, Oxford, 1989).

[52] To correct for the unphysical behavior of the BKS model at short range, we use a potential of the form, $\Phi_{i j}=$ $\Phi_{i j}^{B K S}+4 \epsilon_{i j}\left[\left(\sigma_{i j} / r\right)^{30}-\left(\sigma_{i j} / r\right)^{6}\right]$, where $\Phi_{i j}^{B K S}$ is the original BKS form, and $r$ is the separation between between atoms $i$ and $j$, which may be either Si or O atoms. $\epsilon_{\text {SiSi }}=\sigma_{\text {SiSi }}=0$, while $\epsilon_{\text {SiO }}=4.9634598 \times 10^{-22} \mathrm{~J}$, $\epsilon_{S i O}=0.1313635 \mathrm{~nm}, \epsilon_{O O}=1.6839685 \times 10^{-22} \mathrm{~J}$, and $\sigma_{O O}=0.1779239 \mathrm{~nm} . \sigma_{a b}$ and $\epsilon_{a b}$ are chosen so that the original BKS potential is modified as little as possible at larger $r$, subject to the constraint that the new potential has no inflection at small $r$.

[53] H.B. Callen, Thermodynamics and an Introduction to Thermostatistics, 2nd edition (John Wiley and Sons, New York, 1985).

[54] The curve fitting, differentiations, and integrations required to build the functional model of the thermodynamic properties are carried out using the symbolic computation system Maple, version V.5, Waterloo Maple, Inc., Waterloo, Canada. 


\begin{tabular}{|l|c|c||c|c|c|}
\hline \multicolumn{3}{|c||}{ BKS isochores } & \multicolumn{3}{c|}{ WAC isochores } \\
\hline label & $V\left(\mathrm{~cm}^{3} / \mathrm{g}\right)$ & $\rho\left(\mathrm{g} / \mathrm{cm}^{3}\right)$ & label & $V\left(\mathrm{~cm}^{3} / \mathrm{g}\right)$ & $\rho\left(\mathrm{g} / \mathrm{cm}^{3}\right)$ \\
\hline B1 & 0.4334 & 2.3071 & W1 & 0.5555 & 1.8 \\
B2 & 0.4081 & 2.4501 & W2 & 0.5263 & 1.9 \\
B3 & 0.3828 & 2.6119 & W3 & 0.5000 & 2.0 \\
B4 & 0.3575 & 2.7966 & W4 & 0.4761 & 2.1 \\
B5 & 0.3322 & 3.0093 & W5 & 0.4545 & 2.2 \\
B6 & 0.3070 & 3.2572 & W6 & 0.4347 & 2.3 \\
B7 & 0.2817 & 3.5495 & W7 & 0.4166 & 2.4 \\
B8 & 0.2564 & 3.8994 & W8 & 0.4000 & 2.5 \\
B9 & 0.2311 & 4.3260 & W9 & 0.3846 & 2.6 \\
B10 & 0.2058 & 4.8572 & W10 & 0.3571 & 2.8 \\
& & & W11 & 0.3333 & 3.0 \\
& & & W12 & 0.3125 & 3.2 \\
& & & W13 & 0.2941 & 3.4 \\
& & & W14 & 0.2777 & 3.6 \\
& & & W15 & 0.2500 & 4.0 \\
\hline
\end{tabular}

[55] S. Sastry, P.G. Debenedetti, F. Sciortino, and H.E. Stanley, Phys. Rev. E 53, 6144 (1996). Note that the definition used here for the $K_{T}^{\max }$ line differs from that of the "temperature of extremal compressibility" line studied by these authors. We focus on the $K_{T}^{\max }$ line because it's location would be simpler to identify in isothermal compression experiments.

[56] Th. M. Nieuwenhuizen, Phys. Rev. Lett. 80, 5580 (1998); ibid. 79, 1317 (1997).

[57] F. Sciortino and P. Tartaglia, cond-mat/0007208 (2000).

[58] J. Horbach and W. Kob, Phys. Rev. B 60, 3169 (1999).

[59] C.A. Angell and H. Kanno, Science 193, 1121 (1976); C.A. Angell, P.A. Cheeseman and S. Tamaddam, Science 218, 885 (1982).

[60] R. Bruckner, J. Non-Cryst. Solids 5, 123 (1970).

[61] R.A. Fine and F.J. Millero, J. Chem. Phys. 59, 5529 (1973).

[62] G.P. Johari, A. Hallbrucher and E. Mayer, Nature 330, 552 (1987).

TABLE I: Volume $V$ and density $\rho$ of each isochore simulated for BKS and WAC silica. The labels are used to identify isochores shown in the figures. 


\begin{tabular}{|c|c|c|c|c|}
\hline model & $\mathrm{n}$ & $\alpha_{n}$ & $\beta_{n}$ & $\gamma_{n}$ \\
\hline WAC & 0 & -9.13919 & -0.00627597 & 122.772 \\
$T_{0}=7000 \mathrm{~K}$ & 1 & -38.8581 & 0.108913 & -328.537 \\
& 2 & 163.650 & -0.494217 & 311.922 \\
& 3 & -306.599 & 0.983621 & -328.537 \\
& 4 & 205.422 & -0.683688 & 29.1778 \\
& 5 & - & - & 2.31542 \\
\hline BKS & 0 & -1.01756 & 0.00144202 & 479.401 \\
$T_{0}=5000 \mathrm{~K}$ & 1 & -61.1632 & 0.0488104 & -708.648 \\
& 2 & 293.988 & -0.381123 & 409.488 \\
& 3 & -621.091 & 1.069764 & -117.045 \\
& 4 & 478.866 & -0.969089 & 16.6264 \\
& 5 & - & - & -0.904994 \\
\hline
\end{tabular}

TABLE II: Coefficients of polynomial fits for $V$ dependence of $a$ and $b$; and the $\rho$ dependence of $P$ for $T=T_{0}$. The units for each coefficient are appropriate to give $a$ in $\mathrm{MJ} / \mathrm{mol}, b$ in $\mathrm{MJ} /\left(\mathrm{mol} \mathrm{T}^{3 / 5}\right)$, and $P$ in GPa, when $V$ is given in $\mathrm{cm}^{3} / \mathrm{g}$ and $\rho$ is measured in $\mathrm{g} / \mathrm{cm}^{3}$.

\begin{tabular}{|l|c|c|c|c|c|c|}
\hline & $T^{*}$ & $T_{c}$ & $T_{g}$ & $T_{g} / T^{*}$ & $T_{c} / T^{*}$ & $T_{c} / T_{g}$ \\
\hline WAC & 9000 & 4000 & $<4000$ & $<0.44$ & 0.44 & $>1$ \\
$\mathrm{BKS}$ & 5000 & 2000 & $1380[58$ & 0.28 & 0.40 & 1.45 \\
$\mathrm{ST2}$ & $330[12$ & $235[13$ & $<235$ & $<0.71$ & 0.71 & $>1$ \\
\hline $\mathrm{SiO}_{2}$ & $1823[59$ & $\sim 730$ & $1450[60$ & 0.80 & $\sim 0.40$ & 0.50 \\
$\mathrm{H}_{2} \mathrm{O}$ & $277[61]$ & $\sim 194$ & $136[62]$ & 0.49 & $\sim 0.70$ & 1.4 \\
\hline
\end{tabular}

TABLE III: Comparison of characteristic temperatures of simulated and real tetrahedral liquids. $T^{*}$ for real $\mathrm{SiO}_{2}$ and $\mathrm{H}_{2} \mathrm{O}$ are estimated as the ambient $P$ values of the TMD; the actual value of $T^{*}$ for these systems is likely to be slightly higher. Upper bounds on $T_{g}$ for WAC silica and ST2 water are taken simply as the lowest $T$ at which equilibrium simulations of the liquid have been conducted. Values preceded by " " are found by assuming that the values of $T_{c} / T^{*}$ found from simulations apply to the real substances. References are provided in brackets for those values not determined here. 

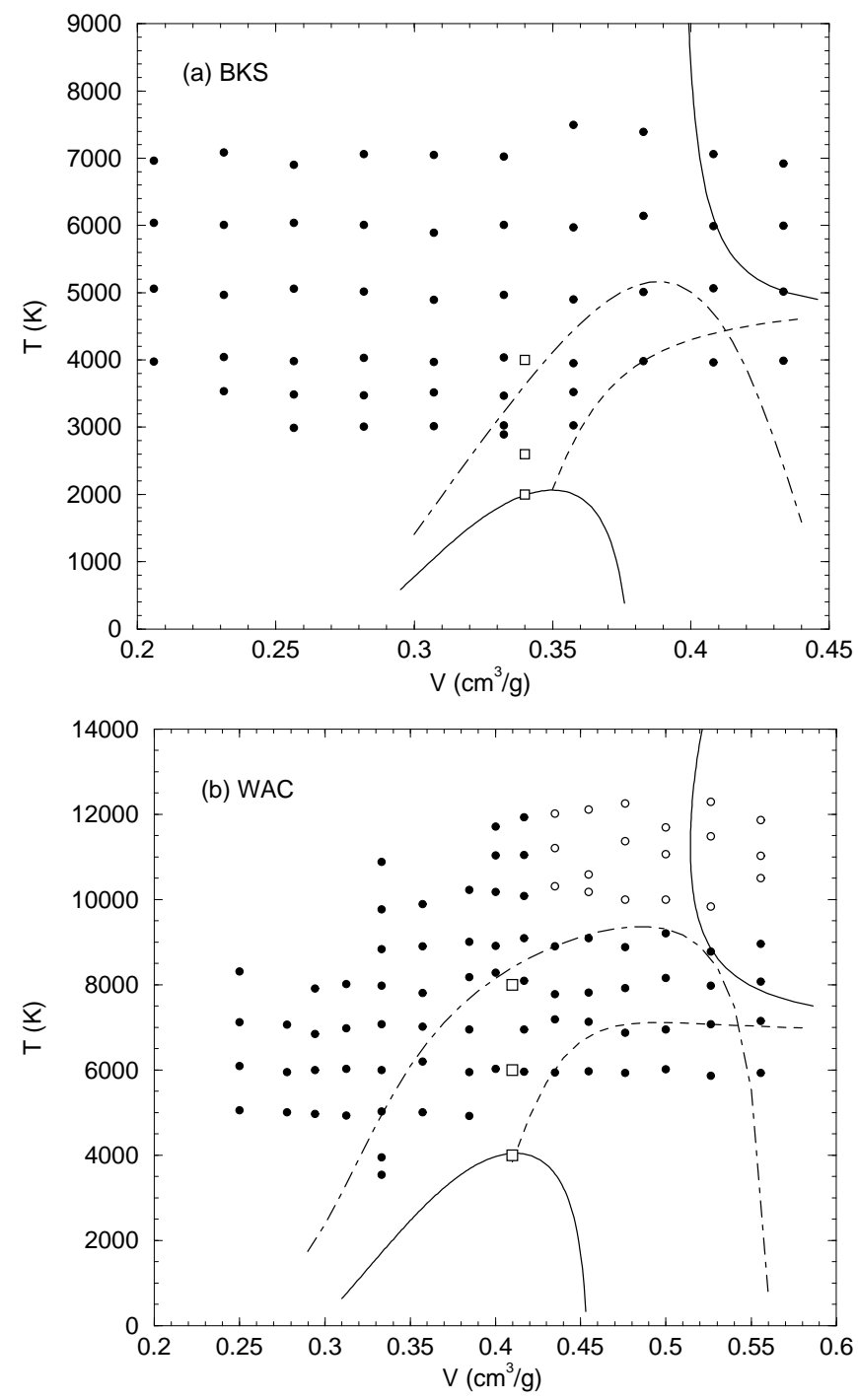

FIG. 1: Simulated state points and thermodynamic features of (a) BKS and (b) WAC silica. The model equations of state derived in Section IV are fit to MD simulation results obtained at the $(V, T)$ points indicated by filled circles. In (b) open circles are WAC state points at which substantial deviations from Eq. 1 are observed, and so are excluded from the data set used to construct the equation of state. The BKS and WAC equations of state give estimates for the projection into the $(V, T)$ plane of the spinodals (solid lines), TMD line (dotdashed), and $K_{T}^{\max }$ line (dashed), as defined in Section $\mathrm{V}$. The open squares locate $(V, T)$ points at which we test for liquid-liquid phase separation, as described in Section VI. 

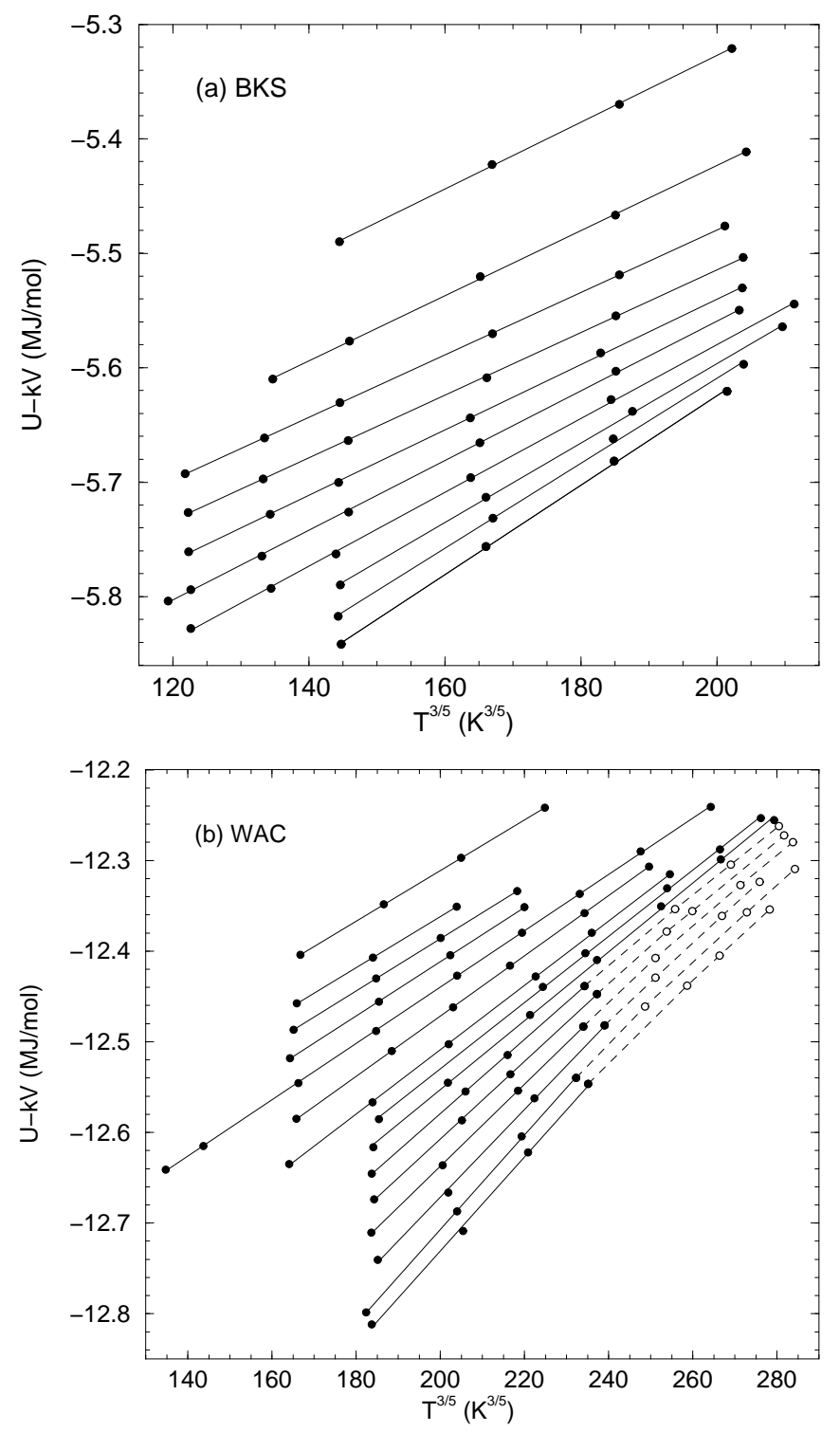

FIG. 2: Isochores of $U$ versus $T^{3 / 5}$ for (a) BKS and (b) WAC silica. Symbols are $U$ values obtained from MD simulation, while solid lines show linear fits to each isochore. To better view each isochore and their fitted lines, we plot $U-k V$, with $k=1(\mathrm{~g} \mathrm{MJ}) /\left(\mathrm{cm}^{3} \mathrm{~mol}\right)$ and $V$ measured in $\mathrm{cm}^{3} / \mathrm{g}$, so that each isochore is subject to a $V$-dependent shift to separate it from the others. In (a) isochores B1 through B10 are shown from bottom to top; in (b) isochores W1 through W15 are shown from bottom to top. Also in (b) are shown high $T$ points (open circles connected by dashed lines) on the largest $V$ isochores which deviate from linear behavior and so are excluded from the fits. The statistical error for the $U$ values shown in (a) and (b) does not exceed $\pm 0.004 \mathrm{MJ} / \mathrm{mol}$. 

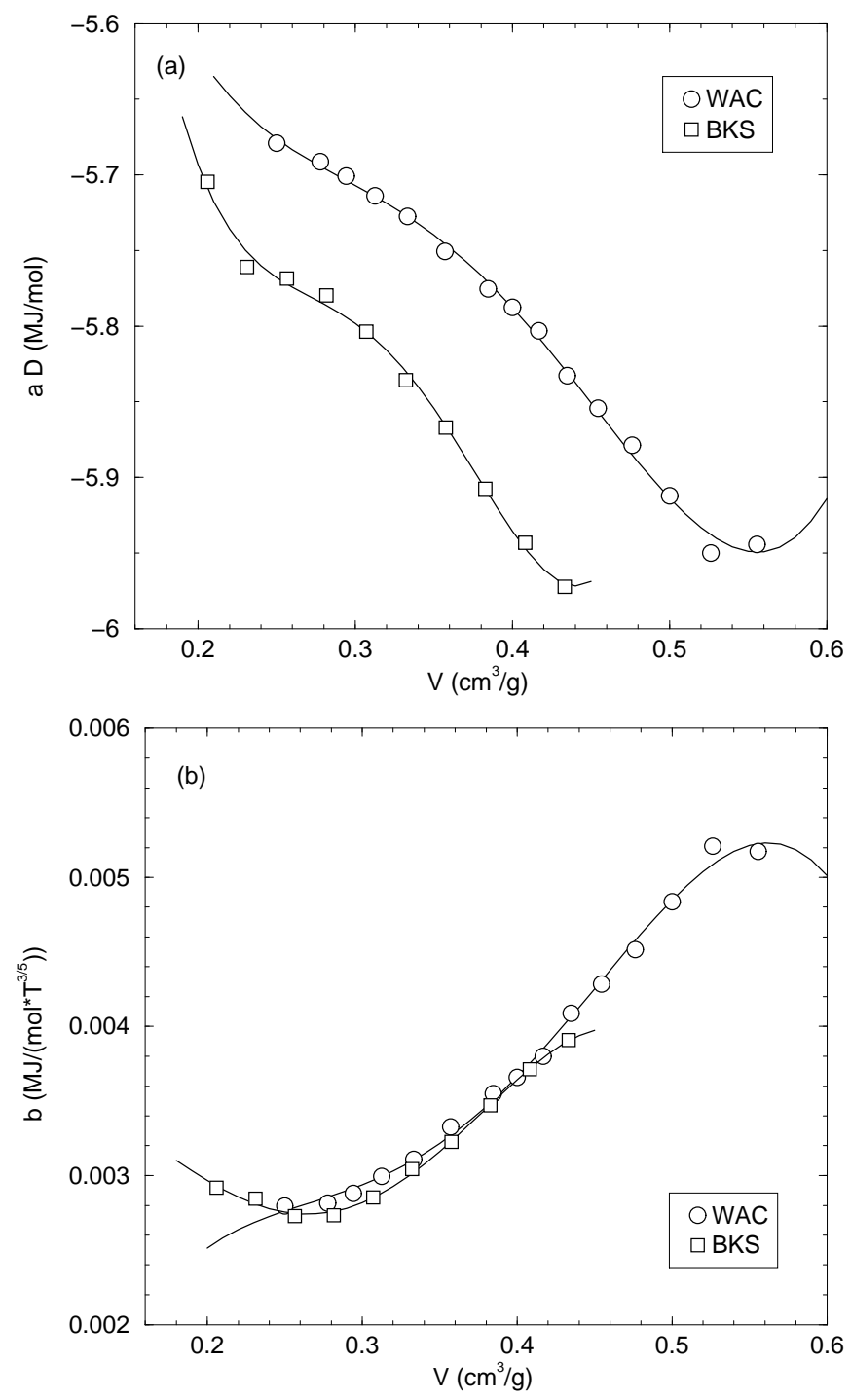

FIG. 3: $V$ dependence of (a) $a$ and (b) $b$ for BKS and WAC silica, found from the linear fits to the isochores shown in Fig. 2. Solid curves are fits to the data of a fourth order polynomial in $V$. In (a) $D$ is a scale factor to permit both curves to be compared in a single plot; for BKS, $D=1$ and for WAC, $D=0.45$. 


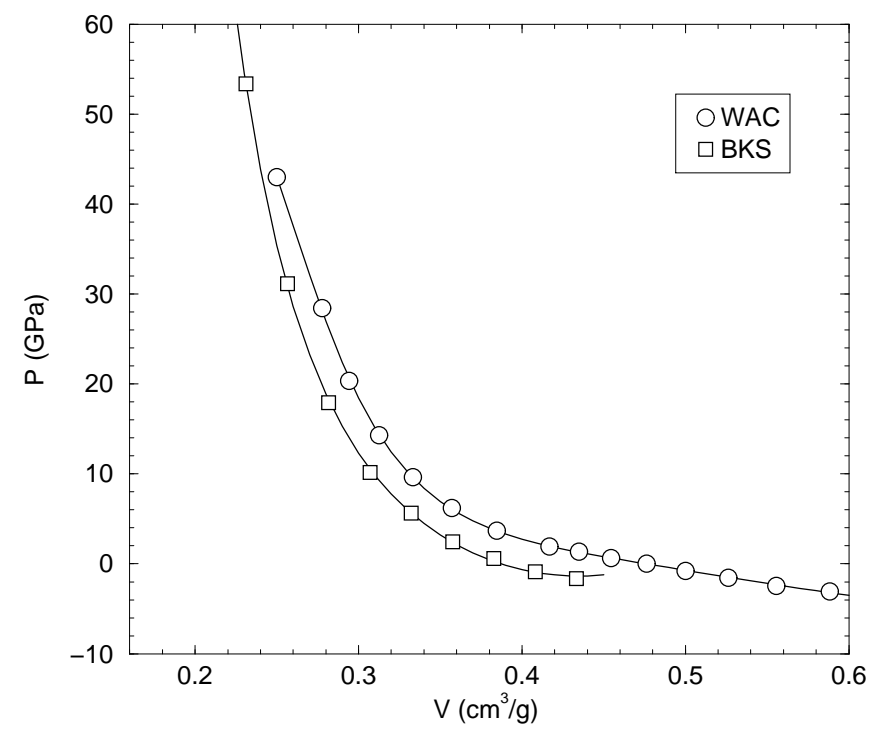

FIG. 4: Isotherms of $P$ versus $V$ for BKS $\left(T_{0}=5000 \mathrm{~K}\right)$ and WAC $\left(T_{0}=7000 \mathrm{~K}\right)$ silica. Solid curves are fits to the data of a fifth order polynomial in $\rho$. 

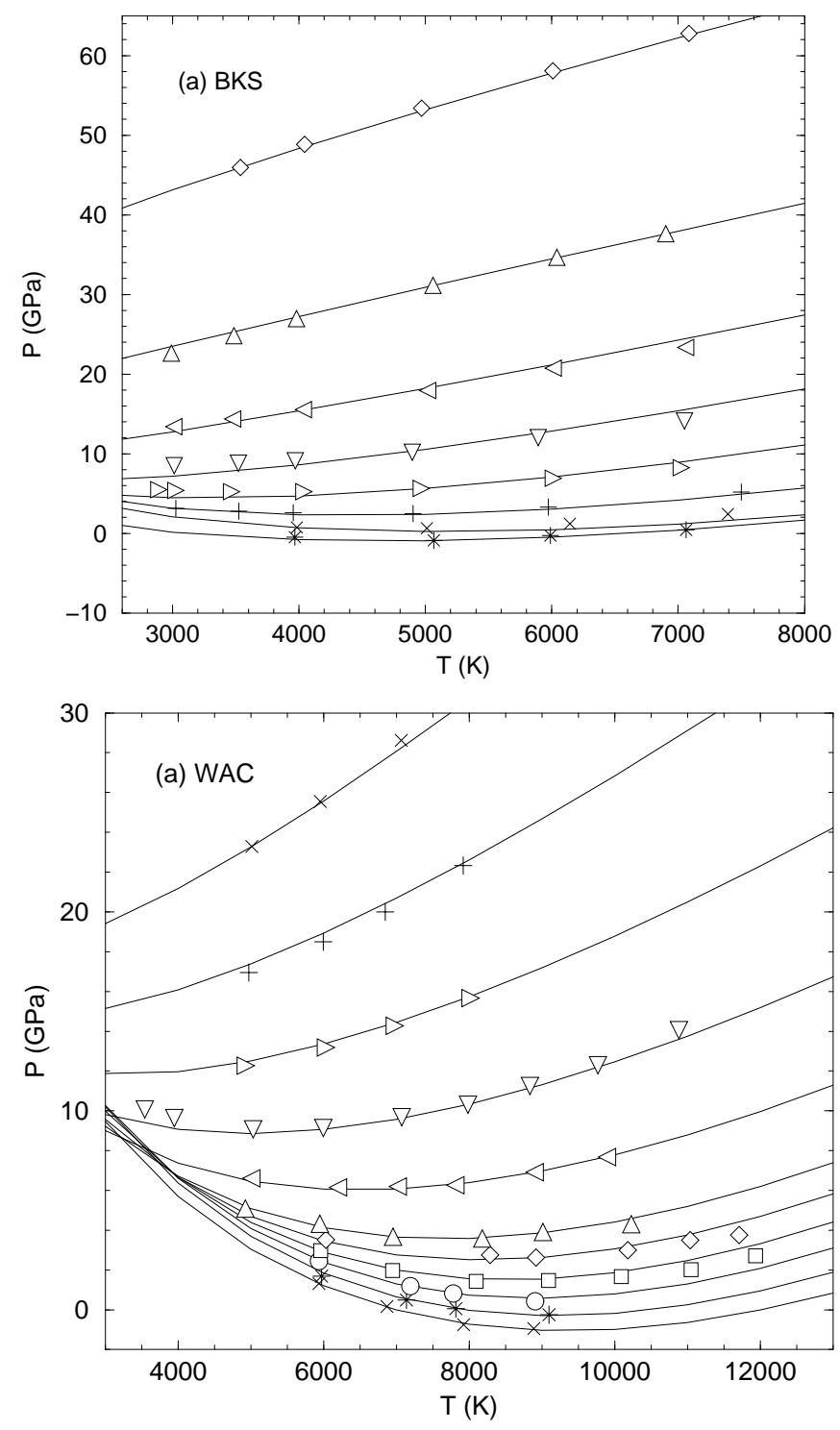

FIG. 5: Isochores of $P$ versus $T$ for (a) BKS and (b) WAC silica. Symbols are values obtained from MD simulation, while lines are determined from the model equations of state developed in Section IV. For BKS, isochores B1 through B8 are shown from bottom to top; for WAC, W4 through W15 are shown, from bottom to top. 

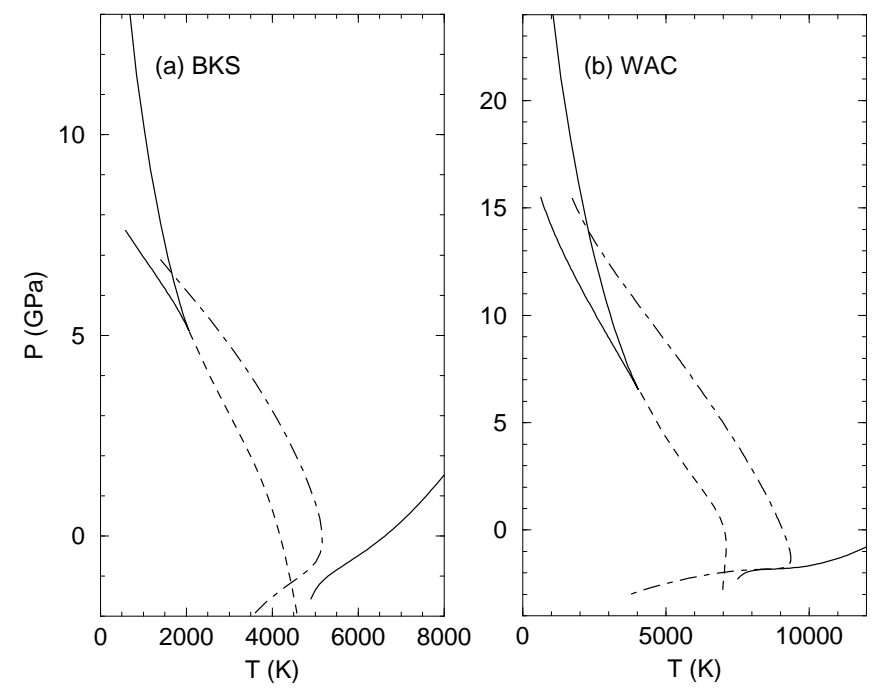

FIG. 6: Estimates for the projection into the $(T, P)$ plane of the spinodals (solid lines), TMD line (dot-dashed), and $K_{T}^{\max }$ line (dashed), evaluated from our model equations of state for (a) BKS and (b) WAC silica.
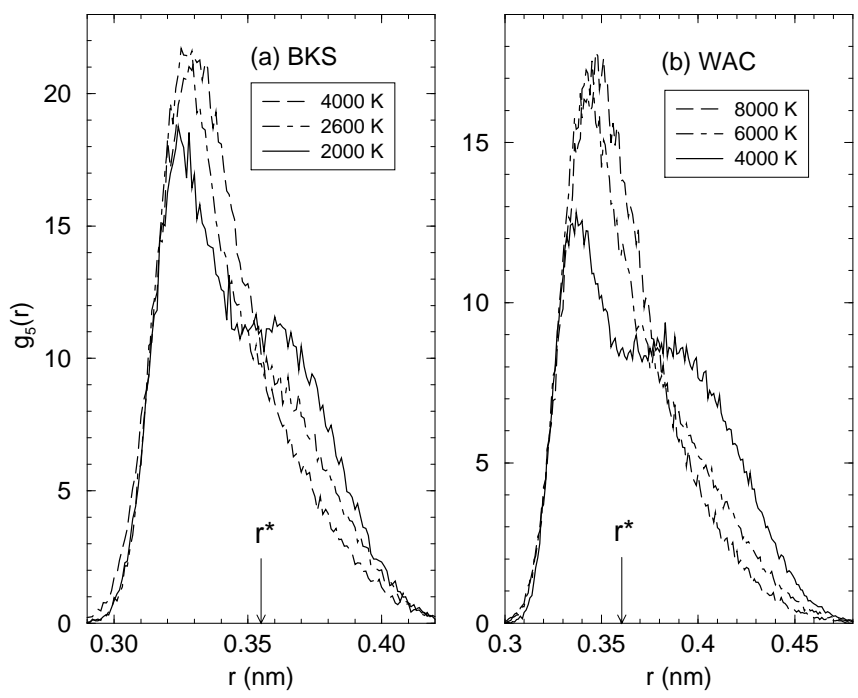

FIG. 7: $g_{5}(r)$ for (a) BKS and (b) WAC silica evaluated at the state points indicated by open squares in Fig. 1. The system size used is $N=750$ atoms. The arrows indicate the values of $r^{*}$ used to identify silicon atoms having highdensity coordination or low-density coordination. For BKS silica, $r^{*}=0.355 \mathrm{~nm}$; for WAC silica, $r^{*}=0.360 \mathrm{~nm}$. 


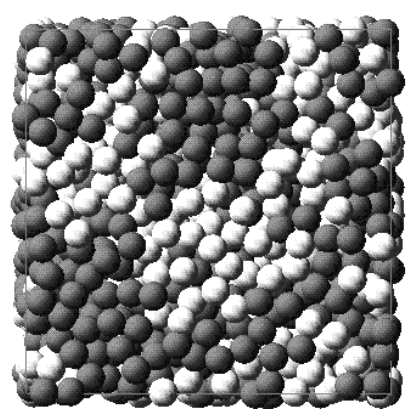

(a) BKS

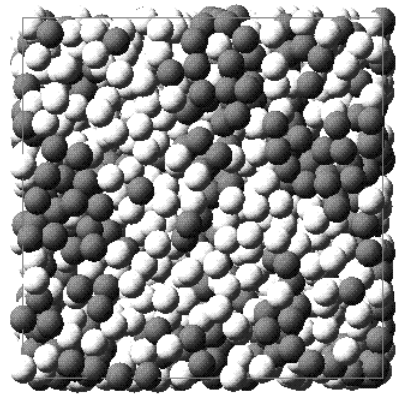

(b) WAC

FIG. 8: Snapshots of MD configurations of BKS silica $(T=$ $\left.2000 \mathrm{~K}, \rho=0.34 \mathrm{~g} / \mathrm{cm}^{3}\right)$ and WAC silica $(T=4000 \mathrm{~K}, \rho=$ $\left.0.41 \mathrm{~g} / \mathrm{cm}^{3}\right)$. The configurations are viewed face-on to one side of the simulation cell. There are $N=6000$ atoms in each system, but only silicon atoms are shown. Dark spheres are silicon atoms having high-density coordination: i.e. a 5th nn silicon within a distance $r<r^{*}$. Light spheres are silicon atoms having low-density coordination: i.e. a 5th nn silicon at a distance $r>r^{*}$. The clustering of atoms with similar coordination into droplets is consistent with the onset of liquid-liquid phase separation. 